\title{
Propagation of Magnetostatic Surface Waves in a Tunable One-Dimensional Magnonic Crystal
}

\author{
M. E. Dokukin, K. Togo and M. Inoue \\ Toyohashi University of Technology, 1-1 Hibari-Ga-Oka, Tempaku, Toyohashi 441-8580, Japan
}

We have studied propagation of magnetostatic surface waves in yttrium iron garnet films with attached 1D periodic arrays of metal strips. For different experimental geometries and different designs of 1D periodic arrays of metal strips, propagation spectra of magnetostatic surface waves were measured and analyzed. We have shown a way to control propagation characteristics of magnetostatic surface waves by changing of electrical contact between periodic metal strips.

Key words: magnonic crystal, magnetostatic surface waves, yttrium iron garnet film

\section{Introduction}

Microwave ferrite-based devices have unique properties for radio-frequency applications. Therefore, it is very important for such devices to have an additional possibility of dynamic control at high frequency. An yttrium iron garnet (YIG) has the most appropriate characteristics for high-frequency range. YIG films support magnetostatic wave (MSW) propagation and widely used as high-frequency MSW filters, delay lines or resonators ${ }^{1)}$. These devices are fabricating in magnetized thin YIG films with attached microstrip antennas. For such devices, the MSW frequency band may be tuned over a range of $1-20 \mathrm{GHz}$. At the change of the magnetic field by tens of Oe, the excitation wave number and group velocity may be changed by order of magnitude. Nowadays more attention has been paid to MSW in periodic structures. In such structures MSW have the same behavior as light in photonic crystal. Analog of a photonic crystal for the case of MSW, was entitled magnonic crystal2). In magnonic crystals, we can control a propagation of magnetostatic waves by forming the periodic structure. As in the case of photonic crystals, the magnonic crystals can be distinguished by dimensionality. The propagation of MSW in ferrite films with different type of $1 \mathrm{D}$ periodicity has been extensively studied for more then thirty years. In particular, Sykes et al. show the magnetostatic wave propagation through a periodic structure formed by selectively surface etching3). Such system exhibits stop bands in MSW propagation spectrum, which appears due to reflection from periodic structural grooves. More detailed study of static 1D periodic structural grating on the YIG film's surface was made by Maeda and Susaki in 20064). In 1977 Owens et al. have been discussed an influence of periodic metal strips array to MSW propagation spectrum and creation of wideband MSW reflector based on parallel aluminum strips ${ }^{5)}$. Interaction of MSW with a periodic and inhomogeneous magnetic field has been intensively studied last fifteen years ${ }^{6)-9}$.
In these works, authors demonstrated the MSW propagation behavior and formation of stop bands in ferrite films spectra, which were magnetized by a modulated periodic field ${ }^{6}$ ) or by field with inhomogeneous banklike profile ${ }^{7)}$. Furthermore, the structure with periodic magnetic disturbance is demonstrated to be MSW's waveguide ${ }^{8}$. In addition, an influence of external periodic dynamic magnetic field, which created by meander-type line, to the MSW propagation was studied by Fetisov ${ }^{9}$.

At present, the interest to the $2 \mathrm{D}$ periodic $\mathrm{MSW}$ structures began to arouse. For example, Nikitov et al. ${ }^{2}$ have studied transmission spectra of the $2 \mathrm{D}$ YIG-based magnonic crystal. In this work, it was shown that creation of $2 \mathrm{D}$ periodic holes array on the surface of YIG crystal also leads to stop bands formation in MSW propagation spectra.

However, in previous works only static structures have been studied2)-5), but it is very important for applications to have a possibility of dynamic control of MSW propagation. Therefore, creation of any dynamically changed periodic structures can give a chance to create controllable MSW devices. This work is concerned with the characteristics of magnetostatic surface waves in YIG thin films with attached 1D periodic metal strips arrays with changeable electric contacts between strips.

\section{Experimental setup and measurement specific}

In present study, we use $14 \mu \mathrm{m}$ YIG film grown epitaxially on gadolinium gallium garnet substrate. Two $80 \mu \mathrm{m}$ wide, $5 \mathrm{~mm}$ long and $18 \mu \mathrm{m}$ thick microstrip line transducers was positioned on the top of the filmstrip for the excitation and detection of spin-wave signals.

The static magnetic field $\mathrm{H}_{0}$ was applied in the plane of the film as shown in Figure 1. This configuration corresponds to magnetostatic surface wave (MSSW) propagation. The periodic metal stripes array and microstrip transducers were formed on the one $\mathrm{Cu}$ covered teflon substrate by photolithographic 
technique. The metal strips width and distance between adjacent strips were $300 \mu \mathrm{m}$. Necessary structure pattern was created from photoresist layer after UV exposure. Finally, transducers and strips were formed by etching in $\mathrm{FeCl}_{3}$ solution.

Figure 1 shows the experimental setup and geometry scheme for transmission- and reflection-type measurements. Figures (I) and (II) show a schematic drawing of the transmission- and reflection-type experimental setup respectively. The geometry scheme of open and short transmission-type experiments are presented on figures (a) and (b). Pictures (c)-(e) show different reflection-type experiment scheme. The distance between two transducers, in transmission-type [figs 1 (a) and (b)] experiment, was $5.5 \mathrm{~mm}$. In reflection-type experiment [figs 1 (c)-(e)], the angle between transducers was 90 degrees. Propagation characteristic was obtained by vector network analyzer Anritsu 37347C.

The MSSW propagation control in the YIG film is realized by electrical shorting of side contacts between parallel strips. The shorted stripe array is presented at Fig. 1 (a) [the open-type lattice at Fig. 1 (b)]. For the reflection-type experiment, the shorted lattice is shown on Fig. 1 (d) and the open lattice on Fig. 1 (e). An external magnetic field during experiment was $100 \mathrm{Oe}$ for transmission and 150 Oe for reflection experiment.

\section{Experimental results and discussions}

The amplitude-frequency transmission characteristics of magnetostatic surface waves in the structures under study were measured in the range from 1.2 to $2.4 \mathrm{GHz}$. Note that, the attenuation of the MSSW's amplitude in studied transmission-type structure was $\sim 10 \mathrm{~dB}$. At the same time, the signal inducing in the receiving antenna was smaller than 50 $\mathrm{dB}$.

For transmission-type structure, the propagation spectra are shown in Fig. 2. One can see the propagation spectrum at $1.67 \mathrm{GHz}$ shows stop band; which exists for the open-type (open circles) and disappears for the shorted-type structure (solid line). The wavelength corresponding to the stop band can be calculated with ${ }^{10)}$ :

$$
\lambda=4 \pi d / \ln \left(\omega_{\mathrm{M}}^{2} /\left(\left(2 \omega_{\mathrm{H}}+\omega_{\mathrm{M}}\right)^{2}-4 \omega^{2}\right)\right),
$$

where $\omega_{\mathrm{H}}=\gamma H_{0}, \quad \omega_{\mathrm{M}}=4 \pi \gamma M, d-$ the YIG film thickness, $\omega-$ MSSW frequency, $H_{0}$ - external magnetic field, $\gamma-$ gyromagnetic constant $(\gamma=2.8$ $\mathrm{MHz} / \mathrm{Oe}$ ) and $4 \pi M$ is the saturation magnetization of YIG film. The calculated wavelength for the stop band at $1.67 \mathrm{GHz}$ is of $\sim 600 \mu \mathrm{m}$, that similarly to the lattice period. The disappearance of the stop band at 1.67 $\mathrm{GHz}$ for the shorted strips array can be explained as follows. When MSSW propagate along the YIG film, it can induce a current flow in the metal strips.

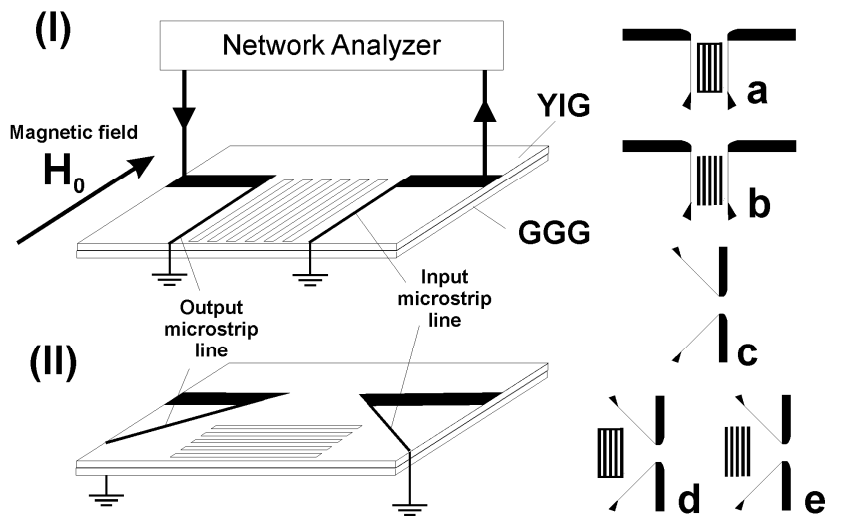

Fig. 1 Experimental setup [figures (I) and (II)] and geometry scheme for open- and short-type periodic metal strips array [figures (a)-(e)]. Figure (I) shows the drawing for the transmission-type experimental setup; figure (II) shows reflection-type setup. In both cases, a magnetic field $\mathrm{HO}$ is applied in the YIG film plane and perpendicular to the long direction of the film strip. Figures (a) and (b) show geometry scheme of open and short transmission-type metal strips array; (d)-(e) - open and short reflection-type metal strips array.

That in case of the open periodic strips array leads to the absorption and scattering of magnetostatic wave energy. However, in the case of the shorted lattice, it leads to occurrence of the back voltage in shorted strips. It is most likely that this back voltage results to suppression of scattering processes and leads MSSW propagation through the structure without attenuation.

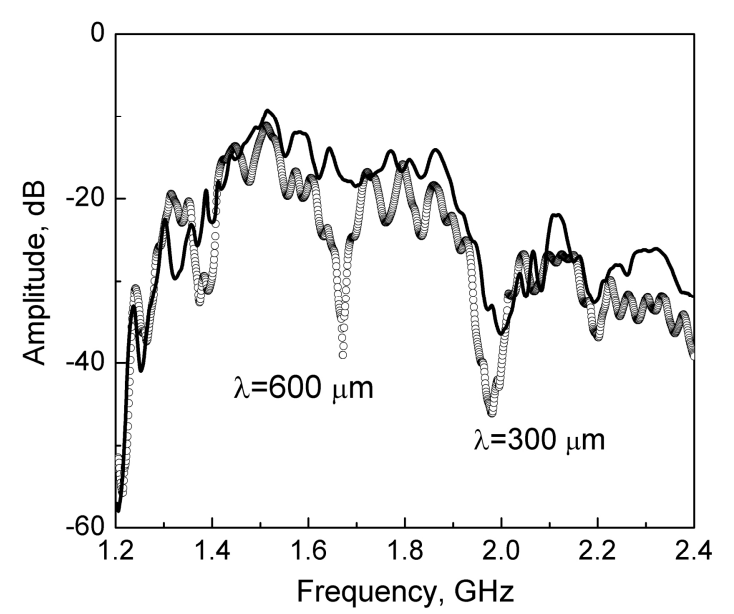

Fig. 2 Amplitude-frequency characteristic of MSSW propagation in the YIG film with attached open [Fig. 1 (b)] (open circles) and short [Fig. 1 (a)] (solid line) periodic strips array (transmission-type experiment). The external magnetic field is 100 Oe. 


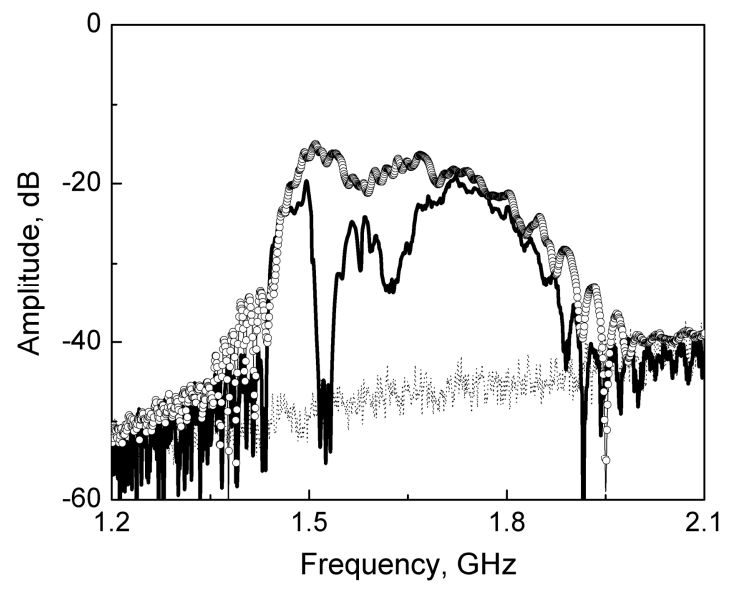

Fig. 3 Amplitude-frequency characteristic of MSSW propagation in the YIG film with attached open [Fig. 1 (e)] (open circles) and short [Fig. 1 (d)] (solid line) periodic strips array (reflection-type experiment). The dotted line gives the spectrum without strips array [Fig. 1 (c)]. The external magnetic field is 150 Oe.

Besides, it is seen that open- and shorted-type structures have a stop band at $2 \mathrm{GHz}$ (the wavelength $\sim 300 \mu \mathrm{m})$. Appearance of this stop band can be explained by reflection of the MSSW from interface between metal covered and metal uncovered YIG film surfaces.

During the propagation along such periodic structure, the MSSW's group velocity is changed, which can lead to scattering of magnetostatic surface waves from metal covered/metal uncovered interfaces.

Moreover, Fig. 2 shows the other stop bands at $1.36 \mathrm{GHz}$ (in the case of open-type array) and $2.2 \mathrm{GHz}$ (in both cases), which agree with Bragg reflection law ( $\lambda=2 \mathrm{~d}$ wavelength condition).

Experimental data for reflection-type structure are presented in Fig. 3. One can see the range of detected signal for both (open and short reflection-type) structures is from 1.4 to $2.0 \mathrm{GHz}$. The amplitude-frequency spectra, which presented in Fig. 3, are corresponded to three reflection-types experimental conditions (see Fig. 1 (c)-(e) for details). The system without array of strips [Fig. 1 (c)] does not show MSSW propagation through the film. It is seen that in this case the level of received signal is $\sim 45 \mathrm{~dB}$ (Fig. 3, dotted line), which can be comparable with electromagnetic noise inducing on receiving antenna. Introducing of the strips array [Fig. 1 (d) and (e)] leads to establishing of MSSW propagation in the structure.

It is seen, that in reflected-type experiment, electrical contacts between metal strips resulted in appearing of stop bands. One can see the first stop band arises at $1.52 \mathrm{GHz}$, and second at $1.62 \mathrm{GHz}$.

As in the case of the transmission-type experiment the appearing of the stop band for shorted strips array is possible to explain by occurrence of back voltage. The shorted structure near $1.52 \mathrm{GHz}$ most likely fulfill the back voltage origin conditions, which result in blocking induced current and moreover lead to block a scattering processes from metal array. This results in appearance of stop band in reflected spectrum.

Thus, in comparison with previous works ${ }^{2)-5}$ it was shown possibility of dynamic switching of stop bands in MSSW propagation spectra by changing of electrical contacts between metal strips. Along with magnetic field-induced MSSW frequency (wavelength) tuning, it gives a wide potential for radio-frequency applications.

\section{Conclusion}

The propagation characteristics of magnetostatic surface waves in the YIG film with attached periodic metal stripes array with changeable electric contacts between strips have been studied. We have considered two different (transmission- and reflection-type) geometries. It was shown that the side shorting of parallel metal strips leads to alter of MSSW propagation conditions and to origin of stop bands in MSSW propagation spectra. It is most likely, that such shorting could lead to back voltage in shorted strips, which results to suppression of scattering processes and leads to MSSW propagation through the structure without attenuation. Thus, creation of the electrical contacts between parallel metal strips gives an opportunity of dynamical control of MSSW propagation along YIG film.

Acknowledgements Authors are grateful to K.H. Shin (Kyungsung University) for helpful advices and S.V. Gerus (Institute of Radio Engineering and Electronics) for providing the YIG samples used in this study.

\section{References}

1) P. Kabos and V. S. Stalmachov, Magnetostatic Waves and Their Application (Chapman \& Hall Press, London, 1994).

2) S. A. Nikitov, C. S. Tsai, Yu. V. Gulyaev, Yu. A. Filimonov, A.I.Volkov, S.L.Vysotskii and Ph.Tailhades: Mater. Res. Soc. Symp. Proc., 834, 87 (2005).

3) C. G. Sykes, J. D. Adam, and J. H. Collins: Appl. Phys. Lett., 29, 388 (1976).

4) A. Maeda and M. Susaki, IEEE Trans. Magn., 42, 3096 (2006).

5) J. M. Owens, J. H. Collins, C. V. Smith, Jr., and I. I. Chiang Appl. Phys. Lett., 31, 781 (1977).

6) A. Yu. Annenkov, S. V. Gerus and I. V. Sotnikov, Journal of Communications Technology and Electronics, 37, 8, 1371 (1992) [in Russian].

7) V. I. Zubkov and V. I. Shcheglov, Tech. Phys. Lett., 27, 348 (2001).

8) A. Yu. Annenkov, S. V. Gerus and S. I. Kovalev, Tech. Phys., 49, 238 (2004)

9) Y. K. Fetisov, N. V. Ostrovskaya and A. F. Popkov, J. Appl. Phys., 79, 5730 (1996).

10) B. Lax and K. Button, Microwave Ferrites and Ferrimagnetics, (McGraw-Hill, New York, 1962).

Received Sep. 07, 2007; Revised Oct. 10, 2007;

Accepted Oct. 12, 2007 